\title{
A SIXTEEN-RELATOR PRESENTATION OF AN INFINITE HYPERBOLIC KAZHDAN GROUP
}

\author{
PIERRE-EMMANUEL CAPRACE
}

\begin{abstract}
We provide an explicit presentation of an infinite hyperbolic Kazhdan group with 4 generators and 16 relators of length at most 73 . That group acts properly and cocompactly on a hyperbolic triangle building of type $(3,4,4)$. We also point out a variation of the construction that yields examples of lattices in $\tilde{A}_{2}$-buildings admitting non-Desarguesian residues of arbitrary prime power order.
\end{abstract}

\section{Hyperbolic KaZhDAN Groups}

On ne peut pas faire plus concis! Raymond Devos, Matière à rire, 1991

The existence of infinite Gromov hyperbolic groups enjoying Kazhdan's property $(\mathrm{T})$ has been known since the origin of the theory of hyperbolic groups, as a combination of the following results.

- Every simple Lie group possesses a cocompact lattice, by [Bor63];

- the rank one simple Lie groups $\operatorname{Sp}(n, 1)$ (with $n \geq 2$ ) and $F_{4}^{-20}$ have (T), by [Kos75] (see also [BdlHV08, §3.3]);

- if a locally compact group $G$ has property $(\mathrm{T})$, then so does every lattice $\Gamma$ in $G$ by [Kaz67] (see also [BdlHV08, Theorem 1.7.1]);

- a cocompact lattice in a rank one simple Lie group is Gromov hyperbolic, since it is virtually the fundamental group of a closed Riemannian manifold of negative sectional curvature, see [Gro87].

The smallest known dimension of a negatively curved closed manifold $M$ such that $\pi_{1}(M)$ has $(\mathrm{T})$ is 8 (namely when $M$ is covered by the symmetric space of $\operatorname{Sp}(2,1)$ ), and I am not aware of any known explicit presentation of the fundamental group $\pi_{1}(M)$ in that case. This is a very interesting and natural problem. By the Hyperbolization Theorem (see AFW15, Theorem 1.7.5] and references therein), the fundamental group of a negatively curved closed manifold $M$ of dimension 1, 2 or 3 is a lattice in $\mathbf{R}$ or $O(2,1)$ or $O(3,1)$. Therefore it cannot be a Kazhdan group by BdlHV08, Theorem 2.7.2] (see also Fuj99 for a more general result on the failure

Date: August 31, 2017.

P.-E.C. is a F.R.S.-FNRS senior research associate, supported in part by EPSRC grant no $\mathrm{EP} / \mathrm{K} 032208 / 1$. 
of property (T) for 3-manifold groups). Whether there exists a negatively curved closed manifold $M$ of dimension 4,5,6 or 7 with a Kazhdan fundamental group is another intriguing open problem. M. Kapovich pointed out to me that the related problem of finding objects of either of the following kinds, is also open:

- a nonpositively curved closed manifold, not homeomorphic to a locally symmetric space, and with a Kazhdan fundamental group;

- a Kazhdan Poincaré duality group not isomorphic to a lattice in a connected Lie group.

The possibility to write down an explicit presentation of an infinite hyperbolic Kazhdan group was first realized in [BS97, Corollary 2], where the geometric approach to property $(\mathrm{T})$ via the spectral gap of finite graphs is exploited (see BdlHV08, Chapter 5] for an exposition of that approach including a historical account). The graphs used in [BS97] are certain Cayley graphs of $\mathrm{SL}_{2}(\mathbf{Z} / n \mathbf{Z})$, which satisfy the required spectral gap condition for $n$ sufficiently large. An alternative source of finite Cayley graphs that enjoy the required spectral condition is suggested by A. Valette in his review of [BS97], but I am not aware of any reference where that suggestion was incarnated into an explicit presentation of a hyperbolic Kazhdan group. A different construction is highlighted by $M$. Bourdon in Bou00, §1.5.3]. It gives rise to cocompact lattices in certain Gromov hyperbolic Fuchsian buildings, and also relies on the geometric approach to property $(\mathrm{T})$. The advantage is that the finite graphs on which the spectral gap condition is tested are finite generalized polygons, and the eigenvalues of their incidence matrix is explicitly known by classical results from [FH64]. Nevertheless, the corresponding group presentations one obtains from that construction take several hundreds relations. The variations on Bourdon's construction described in [Ś01] also seem to require a rather large number of relators. Other examples of infinite hyperbolic Kazhdan groups are studied in [LMW], but no explicit short presentation is recorded there.

Cornelia Drutu asked me whether it was possible to use buildings in order to construct an explicit short presentation of an infinite hyperbolic group with Kazhdan's property (T). As explained in [DK17, Section 19.8]: "while 'generic' finitely presented groups are infinite and satisfy Property (T), finding explicit and reasonably short presentations presents a bit of a challenge". In that context, targeting hyperbolic buildings is especially natural in view of the fact that there exist fiverelator presentations of infinite Kazhdan groups acting properly and cocompactly on buildings of type $\tilde{A}_{2}$, see [Ess13, Examples following Theorem 5.8]. Note that those groups cannot be hyperbolic since they are quasi-isometric to a 2-dimensional Euclidean building. The shortest presentation I could find in attempting to answer her question is the following. 
Theorem 1. The group

$$
\begin{aligned}
E=\langle x, y, z, t, r| & x^{7}, y^{7},[x, y] z^{-1},[x, z],[y, z], \\
& t^{2}, r^{73}, t r t r \\
& {\left[x^{2} y z^{-1}, t\right],\left[x y z^{3}, t r\right],\left[x^{3} y z^{2}, t r^{17}\right], } \\
& {\left[x, t r^{-34}\right],\left[y, t r^{-32}\right],\left[z, t r^{-29}\right], } \\
& {\left.\left[x^{-2} y z, t r^{-25}\right],\left[x^{-1} y z^{-3}, t r^{-19}\right],\left[x^{-3} y z^{-2}, t r^{-11}\right]\right\rangle, }
\end{aligned}
$$

is an infinite Gromov hyperbolic group enjoying Kazhdan's property (T). It acts faithfully, properly, cocompactly (not type-preservingly) on a thick hyperbolic Fuchsian building of type $(3,4,4)$. In particular $E$ is quasi-isometrically rigid by [Xie06].

In view of the relation $[x, y]=z$, the generator $z$ is redundant, and the presentation of $E$ given in Theorem 1 is equivalent to a presentation with 4 generators and 16 relators. This modification increases the length of some of the relators, but one checks that the maximal length of a relator in that 16-relator presentation of $E$ remains equal to 73 .

The group $E$ may be viewed as the fundamental group of a simple complex of finite groups, in the sense of [BH99, Chapter II.12]. The underlying simplicial complex $\mathcal{Y}$ has 11 vertices, denoted by $a, b, c_{1}, \ldots, c_{9}$. It has 19 edges and 9 faces, spanned by $a b c_{i}$ for $i=1, \ldots, 9$. As a metric space, it can be viewed as nine hyperbolic triangles with angles $\pi / 2, \pi / 4, \pi / 6$, glued along their hypothenuse [ab] (the triangle $a b c_{i}$ is depicted in Figure 11). The angle at $a$ is $\pi / 6$ and the angle at $b$ is $\pi / 4$. The vertex group $E_{a}$ is the dihedral group of order 146; in the above presentation of $E$ it is generated by $r$ and $t$. The vertex group $E_{b}$ is the Heisenberg group over $\mathbf{F}_{7}$, of order 343 ; in the presentation above it is generated by $x$ and $y$. The vertex group $E_{c_{i}}$ is the cyclic group of order 14 for $i=1, \ldots, 9$. The edge groups $E_{a c_{i}}$ (resp. $E_{b c_{i}}$ ) are cyclic of order 2 (resp. 7); they are generated by $t, t r, t r^{17}, t^{-34}, t^{-32}, t r^{-29}, t r^{-25}, t r^{-19}$ and $t r^{-11}$ (resp. $x^{2} y z^{-1}, x y z^{3}, x^{3} y z^{2}, x, y, z, x^{-2} y z, x^{-1} y z^{-3}$ and $x^{-3} y z^{-2}$ ). The edge group $E_{a b}$ and the face groups $E_{a b c_{i}}$ are trivial.

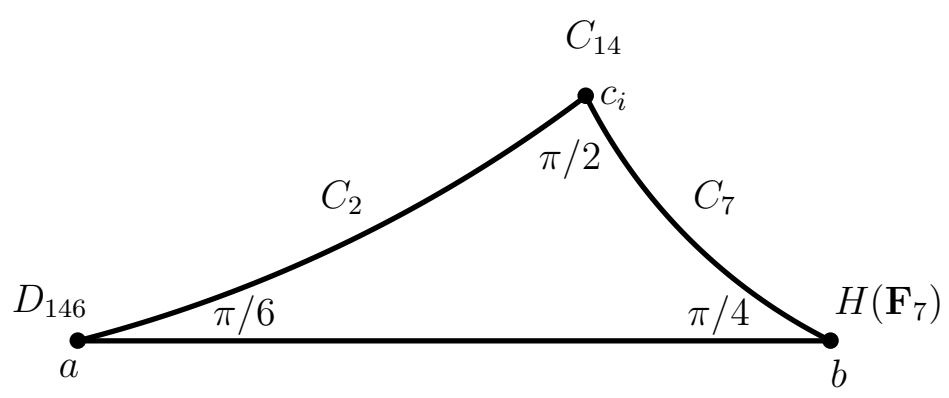

FiguRE 1. The triangle $a b c_{i}$ 
The proof of Theorem 1 consists of the following steps.

- The link at $a$ in the local development of $\mathcal{Y}$ around $a$ is the first barycentric subdivision of the incidence graph of the projective plane over the field $\mathbf{F}_{8}$ of order 8. In particular that link has girth 12, so the Link Condition is satisfied at $a$. This step is achieved by Example 2 and Proposition 3 below.

- The link at $b$ in the local development of $\mathcal{Y}$ around $b$ is the incidence graph of a generalized quadrangle of order $(8,6)$. In particular that link has girth 8 , so the Link Condition is satisfied at $b$. This step is achieved by Corollary 5 below.

- The link at $c_{i}$ is the complete bipartite graph $K_{2,7}$, its girth is 4 , so the Link Condition is satisfied at $c_{i}$ for every $i$.

- By the first three steps, the complex of groups $\mathcal{Y}$ is developable, and its universal cover $\widetilde{\mathcal{Y}}$ is a simplicial complex carrying a $\operatorname{CAT}(-1)$ metric on which the fundamental group $E$ acts faithfully, properly and cocompactly by isometric automorphisms, see [BH99, Theorem II.12.28]. Moreover, since all the links of $\widetilde{\mathcal{Y}}$ are 1-dimensional spherical buildings, it follows from [Tit81, Theorem 1] that $\widetilde{\mathcal{Y}}$ is a 2-dimensional building. It is a hyperbolic Fuchsian building of type $(2,4,6)$ and the $E$-action on it is type-preserving. However $\widetilde{\mathcal{Y}}$ is not thick as a building of type $(2,4,6)$. If one discards the edges of $\widetilde{\mathcal{Y}}$ contained in exactly two 2-simplices, then $\widetilde{\mathcal{Y}}$ becomes a thick building of type $(3,4,4)$ on which $E$ acts in a non-type-preserving way.

- The final step is to check that $E$ has property (T). This is achieved using a straightforward computation based on the criterion established by I. Oppenheim in Opp15, see Proposition 6 below. This completes the proof of Theorem 1 .

\section{ProjeCtive Planes AND DiHEdRAL GROUPS}

We recall that a graph is the incidence graph of a projective plane if and only if it is bipartite, has diameter 3 and girth 6 . We also recall that a difference set in a group $G$ is a subset $\Delta$ of $G$ such that every non-trivial element $g$ of $G$ can be written in a unique way as $g=\sigma^{-1} \tau$ with $\sigma, \tau \in \Delta$. Notice that $G$ must have order $q^{2}+q+1$ where $q=|\Delta|-1$.

Example 2. The set

$$
\begin{aligned}
\Delta & =\{0,1,17,39,41,44,48,54,62\} \\
& =\{0,1,17,-34,-32,-29,-25,-19,-11\}
\end{aligned}
$$

is a difference set in the cyclic group $\mathbf{Z} / 73 \mathbf{Z}$.

Every difference set in a group $G$ gives rise to a projective plane; conversely, every projective plane admitting an automorphism group acting sharply transitively on 
its points gives rise to a difference set. Moreover, for every prime power $q$, the Desarguesian plane of order $q$, i.e. the projective plane over the field $\mathbf{F}_{q}$ of order $q$, has a cyclic automorphism group acting sharply transitively on its points. In particular the cyclic group of order $q^{2}+q+1$ has a difference set. We refer to [BCL, Lemma D.1] and [Dem68, pp. 105-106] for proofs of those assertions.

Given a group $G$ and a collection $\left\{P_{i} \mid i \in I\right\}$ of subgroups of $G$, the coset graph of $G$ with respect to $\left\{P_{i} \mid i \in I\right\}$ is the bipartite graph whose vertex set is the union of $G$ with $\bigcup_{i \in I} G / P_{i}$, and where the element $g \in G$ forms an edge with the coset $h P_{i}$ if and only if $g \in h P_{i}$.

Proposition 3. Let $q$ be a prime power and let $n=q^{2}+q+1$. Let $D_{2 n}=\langle r, t|$ $\left.r^{n}, t^{2}, t r t r\right\rangle$ be the dihedral group of order $2 n$, and let $\Delta$ be a difference set in the cyclic group $\mathbf{Z} / n \mathbf{Z}$. Then:

(i) The Cayley graph of $D_{2 n}$ with respect to the set $\left\{\operatorname{tr}^{\sigma} \mid \sigma \in \Delta\right\}$ is the incidence graph of a projective plane of order $q$.

(ii) The coset graph of $D_{2 n}$ with respect to the subgroups $\left\{\left\langle\operatorname{tr}^{\sigma}\right\rangle \mid \sigma \in \Delta\right\}$ is the first barycentric subdivision of the incidence graph of a projective plane of order $q$.

Proof. Since any reflection in $D_{2 n}$ has non-trivial image in the quotient $D_{2 n} /\langle r\rangle$, it follows that any loop in the Cayley graph $\mathcal{G}$ of $D_{2 n}$ with respect to the set $\left\{\operatorname{tr}^{\sigma} \mid\right.$ $\sigma \in \Delta\}$ has even length. In particular $\mathcal{G}$ is bipartite. If $\mathcal{G}$ contains a loop of length 4 through the identity, then there exist $\sigma_{1}, \ldots, \sigma_{4} \in \Delta$ with $1=\operatorname{tr}^{\sigma_{1}} \operatorname{tr}^{\sigma_{2}} t^{\sigma_{3}} t^{\sigma_{4}}$. Hence $r^{-\sigma_{1}+\sigma_{2}} r^{-\sigma_{3}+\sigma_{4}}=1$. Since $\Delta$ is a difference set, we must have $\sigma_{1}=\sigma_{4}$ and $\sigma_{2}=\sigma_{3}$, so that the loop was a backtracking path. Thus $\mathcal{G}$ has girth at least 6 . Observing that $\mathcal{G}$ is a vertex-transitive bipartite graph of degree $q+1$, we infer that the the total number of vertices at distance exactly 2 from the identity vertex in $\mathcal{G}$ is $q(q+1)$. Since the total number of vertices of $\mathcal{G}$ is $2\left(q^{2}+q+1\right)$ and since $\mathcal{G}$ is bipartite, we deduce that $\mathcal{G}$ has diameter 3 and girth 6 . This proves assertion (i). Assertion (ii) follows from (i) since the coset graph in question is the first barycentric subdivision of $\mathcal{G}$.

\section{Generalized quadrangles and Heisenberg groups}

We recall that a graph is the incidence graph of a generalized quadrangle if and only if it is bipartite, has diameter 4 and girth 8 . The order of a finite generalized quadrangle is the pair $(s, t)$ such that the vertex degrees of the incidence graph of the quadrangle are $s+1$ and $t+1$.

The following observation is closely related to a result of W. Kantor Kan80, Theorem 2]. It allows one to recognize when a coset graph (which is automatically bipartite) is the incidence graph of a generalized quadrangle.

Proposition 4. Let $\mathcal{G}$ be the coset graph of a group $G$ with respect to a collection $\left\{P_{i} \mid i \in I\right\}$ of subgroups.

(i) If $P_{i} \cap P_{j}=\{1\}$ for all distinct $i, j \in I$, then $\mathcal{G}$ has girth $\geq 6$. 
(ii) If $P_{i} P_{j} \cap P_{k}=\{1\}$ for all distinct $i, j, k \in I$, then $\mathcal{G}$ has girth $\geq 8$.

(iii) Let $s=|I|-1$ and suppose that $t=\left|P_{i}\right|-1$ for all $i \in I$. If the condition (ii) holds and if in addition $G$ is finite of order $|G|=(1+t)(1+$ st), then $\mathcal{G}$ is the incidence graph of a generalized quadrangle of order $(s, t)$.

Proof. The proof is a direct computation similar to the proof of Proposition 3 .

The following consequence allows one to recover a family of finite generalized quadrangles that is well-known to the experts; it was first discovered by S. Payne Pay71. The right choice of $p+2$ cyclic subgroups was recorded in [Ess13, Theorem 3.8].

Corollary 5. Let $p$ be an odd prime and $H\left(\mathbf{F}_{p}\right)=\left\langle x, y \mid x^{p}, y^{p},[x, z],[y, z]\right\rangle$ be the Heisenberg group over $\mathbf{F}_{p}$, where $z=[x, y]$. Then the coset graph of $H\left(\mathbf{F}_{p}\right)$ with respect to the collection $\{\langle x\rangle,\langle z\rangle\} \cup\left\{\left\langle x^{a} y z^{-\frac{a}{2}}\right\rangle \mid a=0, \ldots p-1\right\}$ of $p+2$ cyclic subgroups of order $p$ is the incidence graph of a generalized quadrangle of order $(p+1, p-1)$.

Proof. One readily checks that the conditions from Proposition 4 are satisfied.

\section{THE SPECTRAL CRITERION FOR PROPERTY (T)}

The following criterion for property (T) follows easily from the main result of Opp15.

Proposition 6. Let $X$ be a hyperbolic Fuchsian building of type $(3,4,4)$ and $\Gamma$ be a discrete group acting propertly, cocompactly on $X$ by automorphisms. Assume that the projective plane residues of $X$ have order $p+1$ and that the generalized quadrangle residues of $X$ have order $(p+1, p-1)$. If $p \geq 6$, then $\Gamma$ has Kazdhan's property (T).

Proof. We recall from [FH64] that the smallest positive eigenvalue of the Laplacian of the incidence graph of a projection plane of order $p+1$ (resp. a generalized quadrangle of order $(p+1, p-1))$ is $\lambda_{P}=1-\frac{\sqrt{p+1}}{p+2}\left(\operatorname{resp} . \lambda_{Q}=1-\sqrt{\frac{2}{p+2}}\right)$. By Opp15, Theorem 1], the group $\Gamma$ has property $(\mathrm{T})$ provided that the following two conditions hold:

- $\lambda_{P}+2 \lambda_{Q}>3 / 2$,

- $\left(\lambda_{P}+\lambda_{Q}-1\right)^{2}+2\left(\lambda_{P}+\lambda_{Q}-1\right)\left(2 \lambda_{Q}-1\right)>0$.

A straightforward computation shows that the first condition holds for all integer $p \geq 5$, while the second holds for all $p \geq 6$.

\section{VARiations on the SAME Theme}

There is a certain amount of flexibility in the construction of the group $E$ which can be exploited to provide many more infinite hyperbolic Kazhdan groups similar to $E$. The vertex groups $E_{c_{i}}$ need not be cyclic: they could also be chosen to be 
the dihedral group $D_{14}$ of order 14 . One could also permute the edge groups $E_{a c_{i}}$ arbitrarily without changing $E_{b c_{i}}$. The specific choice for the group $E$ in Theorem 1 was made in order to minimize the maximal length of a relation.

Let us note that one can also obtain larger siblings of $E$ as follows. For any Mersenne prime $p$, define a simple complex of groups consisting of $p+2$ hyperbolic triangles of type $(2,4,6)$ glued along their hypothenuse. The two acute vertex groups are a Heisenberg group over $\mathbf{F}_{p}$ and a dihedral group $D_{2 n}$ of order $2 n$, where $n=(p+1)^{2}+p+2$, respectively. The other $p+2$ vertex groups are cyclic or dihedral of order $2 p$. The edge groups are chosen using Proposition 3 and Corollary 5 so that the Link Condition is satisfied at every vertex. We need $p$ to be a Mersenne prime since $p+1$ must be a prime power for Proposition 3 to apply. The fundamental group of that complex is always hyperbolic, and it has property (T) for all $p \geq 7$ by Proposition 6 .

We finish this note by recording another observation that follows from combining Proposition 3 with M. Bourdon's construction from [Bou00, §1.5.3] and its extension due to J. Swiatkowski [Ś

Proposition 7. Let $L$ be the incidence graph of a finite generalized $n$-gon of order $(s, t)$ with $n \geq 3$. Assume that $t$ is a prime power.

Then there is a group $\Gamma$ acting faithfully, properly and cocompactly (but not type preservingly) on a thick locally finite triangle building $X$ of type $(3, n, n)$ admitting $L$ as the link of a vertex.

Proof. We follow the construction described in [ŚS01, §5.3] in order to build $\Gamma$ as the fundamental group of a simple complex of finite groups. The underlying complex $\mathcal{Y}$ is the simplicial cone over the graph $L$. Let $V=V_{1} \cup V_{2}$ be the bipartition of the vertex set of $L$, so that every edge in $L$ joins a vertex in $V_{1}$ to a vertex in $V_{2}$, every vertex in $V_{1}$ has degree $s+1$ and every vertex in $V_{2}$ has degree $t+1$. To each vertex $v$ in $V_{2}$, we define the vertex group $\Gamma_{v}$ as a dihedral group of order $2\left(t^{2}+t+1\right)$. To each edge $e$ belonging to the set $E_{L}(v)$ of edges of $L$ emanating from $v$, we define $\Gamma_{e}$ as a cyclic group of order 2. For all $e \in E_{L}(v)$ we define the inclusion of $\Gamma_{e}$ into $\Gamma_{v}$ in such a way that the coset graph of $\Gamma_{v}$ with respect to $\left\{\Gamma_{e} \mid e \in E_{L}(v)\right\}$ is the first barycentric subdivision of the incidence graph of the Desarguesian projective plane of order $t$. Such a choice is possible in view of Proposition 3 this is where we use the hypothesis that $t$ is a prime power. For $v \in V_{1}$ we define the vertex group $\Gamma_{v}$ to be cyclic of order 2 , and identify $\Gamma_{v}$ with all edge groups $\Gamma_{e}$ with $e \in E_{L}(v)$. The groups attached to all the other simplices of $\mathcal{Y}$ are trivial. By [BH99, Theorem II.12.28], the simple complex of groups defined in this way is developable. By [Tit81, Theorem 1], the univseral cover $\tilde{\mathcal{Y}}$ is a non-thick triangle building of type $(2,6, n)$. Upon discarding the edges of $\tilde{\mathcal{Y}}$ that cover edges of $L$, we may view $\tilde{\mathcal{Y}}$ is a thick triangle building of type $(3, n, n)$ on which $\Gamma$ acts faithfully, properly and cocompactly, but not type-preservingly. 
The difference between Bourdon's construction [Bou00, §1.5.3] and Proposition 7 is that the former yields Fuchsian buildings of type $(2, n, n)$.

Remark 8. Proposition 7 comes close to a solution of a problem posed by W. Kantor [Kan86, Problem C.6.7]. It notably implies that, all finite projective planes satisfying the Prime Power Conjecture appear as residue planes in $\tilde{A}_{2}$-buildings admitting a discrete cocompact group of automorphisms. In particular, all known non-Desarguesian finite projective planes do. This provides a construction of an infinite family of cocompact lattices in exotic $\tilde{A}_{2}$-buildings of arbitrarily large thickness, where exotic means non-isomorphic to the Bruhat-Tits building of a simple algebraic group over a local field. In particular, the main result of [BCL] applies to those lattices, which ensures that they do not admit any finite-dimensional representation with infinite image over any field. The first construction of an infinite family of cocompact lattices in exotic $\tilde{A}_{2}$-buildings was obtained in [BCL, Appendix D]; since then another source of cocompact lattices in exotic $\tilde{A}_{2}$-buildings of arbitrarily large thickness has been identified by N. Radu [Rad17b]. The first example of a cocompact lattice in an $\tilde{A}_{2}$-building admitting non-Desarguesian residue planes is due to him Rad17a]. That example remains the only known $\tilde{A}_{2}$-building with a cocompact lattice where all residue planes are non-Desarguesian.

\section{ACKNOWLEDGEMENTS}

I thank the Isaac Newton Institute for Mathematical Sciences, Cambridge for support and hospitality during the programme Non-positive curvature group actions and cohomology where part of the work on this paper was accomplished. I am grateful to Cornelia Drutu for asking me the question that initiated this note, to Marc Bourdon for explaining me his inspiring construction from [Bou00, §1.5.3], and to U. Bader, M. Kapovich and H. Wilton for their comments on an earlier version of the manuscript.

\section{REFERENCES}

[AFW15] Matthias Aschenbrenner, Stefan Friedl, and Henry Wilton, 3-manifold groups, EMS Series of Lectures in Mathematics, European Mathematical Society (EMS), Zürich, 2015. MR 34441871

[BCL] Uri Bader, Pierre-Emmanuel Caprace, and Jean Lécureux, On the linearity of lattices in affine buildings and ergodicity of the singular cartan flow, Preprint, arXiv:1608.06265. 5, 8

[BdlHV08] Bachir Bekka, Pierre de la Harpe, and Alain Valette, Kazhdan's property (T), New Mathematical Monographs, vol. 11, Cambridge University Press, Cambridge, 2008. MR 24158341, 2

[BH99] Martin R. Bridson and André Haefliger, Metric spaces of non-positive curvature, Grundlehren der Mathematischen Wissenschaften [Fundamental Principles of Mathematical Sciences], vol. 319, Springer-Verlag, Berlin, 1999. MR 17444866 3, 4 ,

[Bor63] Armand Borel, Compact Clifford-Klein forms of symmetric spaces, Topology 2 (1963), 111-122. MR 0146301 1 
[Bou00] Marc Bourdon, Sur les immeubles fuchsiens et leur type de quasi-isométrie, Ergodic Theory Dynam. Systems 20 (2000), no. 2, 343-364. MR 1756974 2, 7, 8

[BS97] W. Ballmann and J. Światkowski, On $L^{2}$-cohomology and property (T) for automorphism groups of polyhedral cell complexes, Geom. Funct. Anal. 7 (1997), no. 4, 615-645. MR 14655982

[Dem68] P. Dembowski, Finite geometries, Ergebnisse der Mathematik und ihrer Grenzgebiete, Band 44, Springer-Verlag, Berlin-New York, 1968. MR 0233275 5

[DK17] Cornelia Drutu and Michael Kapovich, Geometric group theory, Book to appear, 2017. 2

[Ess13] Jan Essert, A geometric construction of panel-regular lattices for buildings of types $\tilde{A}_{2}$ and $\tilde{C}_{2}$, Algebr. Geom. Topol. 13 (2013), no. 3, 1531-1578. MR 3071135 2, 6

[FH64] Walter Feit and Graham Higman, The nonexistence of certain generalized polygons, J. Algebra 1 (1964), 114-131. MR 01709552, 6

[Fuj99] Koji Fujiwara, 3-manifold groups and property $T$ of Kazhdan, Proc. Japan Acad. Ser. A Math. Sci. 75 (1999), no. 7, 103-104. MR 17298531

[Gro87] M. Gromov, Hyperbolic groups, Essays in group theory, Math. Sci. Res. Inst. Publ., vol. 8, Springer, New York, 1987, pp. 75-263. MR 919829 1

[Kan80] William M. Kantor, Generalized quadrangles associated with $G_{2}(q)$, J. Combin. Theory Ser. A 29 (1980), no. 2, 212-219. MR 583960 5

[Kan86]_, Generalized polygons, SCABs and GABs, Buildings and the geometry of diagrams (Como, 1984), Lecture Notes in Math., vol. 1181, Springer, Berlin, 1986, pp. 79158. MR 8433908

[Kaz67] D. A. Kazhdan, On the connection of the dual space of a group with the structure of its closed subgroups, Funkcional. Anal. i Priložen. 1 (1967), 71-74. MR 02093901

[Kos75] Bertram Kostant, On the existence and irreducibility of certain series of representations, 231-329. MR 03993611

[LMW] Alex Lubotzky, Jason Manning, and Henry Wilton, Generalized triangle groups, expanders, and a problem of Agol and Wise, Preprint, arXiv:1702.08200. 2

[Opp15] Izhar Oppenheim, Property (T) for groups acting on simplicial complexes through taking an "average" of Laplacian eigenvalues, Groups Geom. Dyn. 9 (2015), no. 4, 11311152. MR 34284104,6

[Pay71] Stanley E. Payne, Nonisomorphic generalized quadrangles, J. Algebra 18 (1971), 201212. MR 02796726

[Rad17a] Nicolas Radu, A lattice in a residually non-Desarguesian $\tilde{A}_{2}$-building, Bull. Lond. Math. Soc. 49 (2017), no. 2, 274-290. MR 3656296 8

[Rad17b] , PhD thesis, in preparation, 2017. 8

[Ś01] Jacek Światkowski, Some infinite groups generated by involutions have Kazhdan's property (T), Forum Math. 13 (2001), no. 6, 741-755. MR 18612472, 7

[Tit81] J. Tits, A local approach to buildings, The geometric vein, Springer, New York-Berlin, 1981, pp. 519-547. MR 6618014.7

[Xie06] Xiangdong Xie, Quasi-isometric rigidity of Fuchsian buildings, Topology 45 (2006), no. 1, 101-169. MR 21704963

Université Catholique de Louvain, iRmp, Chemin du Cyclotron 2, Bte L7.01.02, 1348 Louvain-la-Neuve, Belgique

E-mail address: pe.caprace@uclouvain.be 